\title{
MelaNostrum: a consensus questionnaire of standardized epidemiologic and clinical variables for melanoma risk assessment by the melanostrum consortium
}

\author{
Alexander J. Stratigos, ${ }^{1, *, \dagger}$ Maria Concetta Fargnoli, ${ }^{2, \dagger}$ Arcangela De Nicolo, ${ }^{3, \dagger}$ Ketty Peris, ${ }^{4}$ \\ Susana Puig, ${ }^{5,6,7}$ Efthymia Soura, ${ }^{1}$ Chiara Menin, ${ }^{8}$ Donato Calista, ${ }^{9}$ Paola Ghiorzo, ${ }^{10}$ Mario Mandala, ${ }^{11}$ \\ Daniela Massi, ${ }^{12}$ Monica Rodolfo, ${ }^{13}$ Laura Del Regno, ${ }^{4}$ Irene Stefanaki, ${ }^{1}$ Helen Gogas, ${ }^{14}$ \\ Veronique Bataille, ${ }^{15}$ Margaret A. Tucker, ${ }^{16}$ David Whiteman, ${ }^{17}$ Eduardo Nagore, ${ }^{18}$ Maria Teresa Landi ${ }^{16}$ \\ ${ }^{1}$ First Department of Dermatology, National and Kapodistrian University of Athens School of Medicine, Andreas Sygros Hospital, \\ Athens, Greece \\ ${ }^{2}$ Department of Dermatology, University of L'Aquila, L'Aquila, Italy \\ ${ }^{3}$ Cancer Genomics Program, Veneto Institute of Oncology IOV - IRCCS, Padua, Italy \\ ${ }^{4}$ Institute of Dermatology, Catholic University, Rome, Italy \\ ${ }^{5}$ Dermatology Department, Melanoma Unit, Hospital Clinic de Barcelona, Universitat de Barcelona, Barcelona, Spain \\ ${ }^{6}$ Instituto de Investigacion Biomedica August Pi i Sunyer (IDIBAPS), Barcelona, Spain \\ ${ }^{7}$ Centro de Investigación Biomédica en Red de Enfermedades Raras (CIBERER), Barcelona, Spain \\ ${ }^{8}$ Immunology and Diagnostic Molecular Oncology Unit, Veneto Institute of Oncology IOV - IRCCS, Padua, Italy \\ ${ }^{9}$ Dermatology Unit, Maurizio Bufalini Hospital, Cesena, Italy \\ ${ }^{10}$ Department of Internal Medicine and Medical Specialties, University of Genoa and Genetics of Rare Cancers, University Hospital \\ Policlinico San Martino-IRCCS, Genoa, Italy \\ ${ }^{11}$ Unit of Melanoma, Department of Oncology and Hematology, Papa Giovanni XXIII Cancer Center Hospital, Bergamo, Italy \\ ${ }^{12}$ Department of Surgery and Translational Medicine, University of Florence, Florence, Italy \\ ${ }^{13}$ Immunotherapy Unit, Department of Experimental Oncology and Molecular Medicine, Fondazione IRCCS Istituto Nazionale dei \\ Tumori, Milan, Italy \\ ${ }^{14}$ Department of Internal Medicine, Laikon Hospital, National and Kapodistrian University of Athens School of Medicine, Athens, \\ Greece \\ ${ }^{15}$ Department of Twin Research and Genetic Epidemiology, Kings College, London, UK \\ ${ }^{16}$ Human Genetics Program, Division of Cancer Epidemiology and Genetics, National Cancer Institute, National Institutes of Health, \\ Bethesda, MD, USA \\ ${ }^{17}$ Population Health Department, QIMR Berghofer Medical Research Institute, Brisbane, Qld, Australia \\ ${ }^{18}$ Department of Dermatology, Instituto Valenciano de Oncología, València, Spain \\ ${ }^{*}$ Correspondence: A. J. Stratigos. E-mail: alstrat2@gmail.com
}

\begin{abstract}
Background Many melanoma observational studies have been carried out across different countries and geographic areas using heterogeneous assessments of epidemiologic risk factors and clinical variables.

Aim To develop a consensus questionnaire to standardize epidemiologic and clinical data collection for melanoma risk assessment.

Methods We used a stepwise strategy that included: compilation of variables from case-control datasets collected at various centres of the MelaNostrum Consortium; integration of variables from published case-control studies; consensus discussion of the collected items by MelaNostrum members; revision by independent experts; addition of online tools and image-based charts; questionnaire testing across centres and generation of a final draft.

Results We developed a core consensus questionnaire (MelanoQ) that includes four separate sections: A. general and demographic data; B. phenotypic and ultraviolet radiation exposure risk factors and lifestyle habits; C. clinical examination, medical and family history; and D. diagnostic data on melanoma (cases only). Accompanying online tools, informative tables, and image-based charts aid standardization. Different subsections of the questionnaire are designed for selfadministration, patient interviews performed by a physician or study nurse, and data collection from medical records.

Conclusions The MelanoQ questionnaire is a useful tool for the collection and standardization of epidemiologic and clinical data across different studies, centres, cultures and languages. This will expedite ongoing efforts to compile highquality data for pooled analyses or meta-analyses and offer a solid base for the design of clinical, epidemiologic and translational studies on melanoma.
\end{abstract}


Received: 3 April 2018; Accepted: 12 July 2018

\section{Conflict of interest}

None declared.

\section{Funding sources}

Funding regarding the Department of Internal Medicine and Medical Specialties, University of Genoa and Genetics of Rare Cancers, Ospedale Policlinico San Martino, Genoa: Italian Ministry of Health 5x1000 per la Ricerca Corrente to Ospedale Policlinico San Martino and AIRC IG 15460. The research at the Melanoma Unit in Barcelona is partially funded by the Spanish Fondo de Investigaciones Sanitarias grants PI15/00716 and PI15/ 00956 co-financed by FEDER 'Una manera de hacer Europa'; CIBER de Enfermedades Raras of the Instituto de Salud Carlos III, Spain, co-financed by European Development Regional Fund 'A way to achieve Europe' ERDF; AGAUR 2014_SGR_603 of the Catalan Government, Spain; European Commission under the 6th Framework Programme, Contract No. LSHC-CT-2006-018702 (GenoMEL) and by the European Commission under the 7th Framework Programme, Diagnoptics; a grant from ‘Fundació La Marató de TV3' 201331-30, Catalonia, Spain; a grant from ‘Fundación Científica de la Asociación Española Contra el Cáncer' GCB15152978SOEN, Spain, and CERCA Programme/Generalitat de Catalunya. Melanoma research at the Department of Dermatology, University of L'Aquila, Italy, was supported by a grant from the Italian Ministry of the University and Scientific Research (PRIN-2012 grant 2012JJX494). MTL and MAT were supported by the Intramural programme of the National Cancer Institute, NIH.

\section{Introduction}

The incidence of cutaneous melanoma (CM) has steadily and significantly increased in populations of European descent in recent years, ${ }^{1}$ including the Mediterranean populations. ${ }^{2-4} \mathrm{CM}$ is responsible for the largest proportion $(60-80 \%)$ of skin cancer-related deaths. ${ }^{5}$ In 2012, more than 232000 people worldwide were diagnosed with CM and more than 55400 succumbed to the disease. ${ }^{5,6}$ Data from population-based European registries have shown an increased incidence of in situ and invasive melanoma but a stable mortality rate in Italy and Spain between 1995 and 2012. ${ }^{7}$ Melanoma risk factors include ultraviolet (UV) radiation exposure, positive family history for melanoma, phenotypic traits with a strong genetic component such as red or blonde hair, light-coloured eyes, and fair skin complexion, and an increased number of common and atypical melanocytic nevi. Even though the advent of targeted therapies and immune checkpoint inhibitors has improved melanoma prognosis in a subgroup of patients, the mortality risk and the socio-economic burden associated with advanced disease emphasize the importance of prevention strategies in high-risk individuals and of screening programs for early detection.

Numerous case-control studies have been carried out focusing on the environmental and phenotypical risk factors of $\mathrm{CM}^{8-16}$ The variables assessed were often heterogeneous, and this has limited the possibility of pooled analyses. Tools for standardized

${ }^{\dagger}$ Contributed equally to this study and share first authorship. A complete list of members of the MelaNostrum Consortium is provided in the Table S1. data recording are therefore needed to design prospective studies and to guide retrieval of previously collected data across various centres. Earlier questionnaires or surveys in melanoma studies focused only on few variables or lacked high test-retest reliability. ${ }^{17-21}$ We sought to generate a comprehensive tool that would allow us to pool data from studies on CM carried out by the MelaNostrum Consortium as well as by other centres.

MelaNostrum is an international Consortium of researchers and clinicians involved in melanoma research in Mediterranean (i.e. Southern European) populations, which have been, so far, under-represented in studies of CM. Formally established in 2017, MelaNostrum comprises experts in various disciplines, including epidemiologists, dermatologists, medical oncologists, pathologists, molecular biologists, geneticists and statisticians. The primary goals of MelaNostrum were as follows: (i) to identify genetic, environmental and phenotypic features associated with melanoma risk; (ii) to investigate disease development and clinical outcomes using molecular classification of melanoma and of melanoma precursors and (iii) to study the role of genetics and immunity in melanoma progression and response to treatment. Currently, MelaNostrum collects and analyzes data and biological samples from 12 centres across Greece, Italy and Spain. A complete list of current MelaNostrum centres and members is provided as Table S1.

Given the heterogeneous recording of variables across melanoma studies, the MelaNostrum investigators were confronted with the difficult task of harmonizing epidemiologic and clinical information from each centre to perform association studies. The present work reports on the stepwise strategy employed to 
develop a data collection tool (hereafter named as MelanoQ) that could be used as a full or itemized template for the standardization of retrospective data from case-control and other epidemiology studies or in prospective studies aimed at the investigation of genetic, lifestyle, epidemiologic and clinical factors associated with melanoma.

\section{Material and methods}

\section{Development of the questionnaire}

The questionnaire was developed in 7 steps as outlined in Fig. 1.

Step 1: compilation of variables from case-control datasets collected by the MelaNostrum centres. Step 2: distribution of the variable list to MelaNostrum members, initial evaluation of differences and similarities across centres and categorization of the items in sections. This step also included enrichment of the variables based on published case-control studies. Step 3: two face-to-face meetings of the MelaNostrum clinical core of investigators, during which each variable in the list was discussed. The first meeting also involved physical examination of patients to achieve consensus on criteria for nevus count and (other) pigmented skin lesion diagnosis. As a result, a draft of the MelanoQ was generated (Barcelona, Spain) and subsequently improved (Athens, Greece). Step 4: review of the draft questionnaire by an independent expert panel (MT, DW, VB) and implementation of the suggested modifications. Step 5: addition of (i) online tools to ease recording of residency and occupations, (ii) tables with lists of ethnicities, outdoor occupations and recreational activities, and (iii) image-based charts for data standardization pertaining to eye and hair colour, tanning ability, freckles and nevi number. Step 6: questionnaire testing across centres, using different languages and recording the time required for completion. Step 7: establishment of the final draft after consensus discussion through email exchange by the MelaNostrum team.

\section{Results}

MelanoQ is organized in 4 main sections (A-D) and includes a total number of 64 items related to: general and demographic information (section A); phenotypic, UV exposure risk factors and lifestyle habits (section B); clinical examination, medical history and family history (section C); tumour characteristics, including histology, staging and molecular profile (section D). Only sections A, B and C are included in the MelanoQ for controls. In each section, the items are formulated as closed-ended questions with only few exceptions. Different subsections are designed for self-administration, patient/control interviews performed by a physician or study nurse, and data collection from medical records. The questionnaire is briefly outlined in Table 1, while it is provided in full as Table S2.

Section A should be filled in by the physician or study nurse (subsection I) and by the study participant (subsection II). In subsection I, the date of questionnaire administration and

\begin{tabular}{|c|c|}
\hline Step 1. & $\begin{array}{l}\text { Collection of variables from case/control datasets } \\
\text { of the MelaNostrum clinical centers }\end{array}$ \\
\hline \multicolumn{2}{|r|}{$\downarrow$} \\
\hline Step 2. & $\begin{array}{l}\text { Distribution of preliminary list of variables to MelaNostrum members for } \\
\text { evaluation, categorization, and enrichment by literature review }\end{array}$ \\
\hline \multicolumn{2}{|r|}{$\begin{aligned} \\
\nabla\end{aligned}$} \\
\hline Step 3. & $\begin{array}{l}\text { Face-to-face meetings }{ }^{\star} \text { to discuss each variable and } \\
\text { development of a draft questionnaire }\end{array}$ \\
\hline \multicolumn{2}{|r|}{$\nabla$} \\
\hline Step 4. & $\begin{array}{l}\text { Assessment of the questionnaire by } \\
\text { an independent expert panel }\left.\right|^{\star *}\end{array}$ \\
\hline \multicolumn{2}{|r|}{$\nabla$} \\
\hline \multicolumn{2}{|r|}{ Addition of online tools, tables, and image-based charts } \\
\hline \multicolumn{2}{|r|}{ 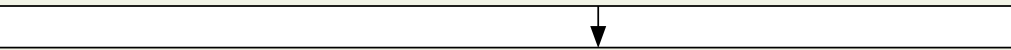 } \\
\hline \multicolumn{2}{|r|}{ Questionnaire testing across centers } \\
\hline \multicolumn{2}{|r|}{$\nabla$} \\
\hline Step 7. & Establishment of the final consensus questionnaire \\
\hline
\end{tabular}

Figure 1 Stepwise strategy used to develop the MelanoQ. *one meeting including patient examination to define criteria for pigmented lesion assessment and nevus count. **The expert panel consisted of M. Tucker (USA), V. Bataille (UK) and D. Whiteman (Australia). 
Table 1 Design and variables of MelanoQ.

\begin{tabular}{|c|c|c|c|}
\hline Section & Completed by & Variables & Items \\
\hline \multicolumn{4}{|l|}{ SECTION A (case/control) } \\
\hline General & Physician/study nurse & Subject ID, date and update of questionnaire, sporadic/familial case & $1-5$ \\
\hline Demographic & Self-administered & $\begin{array}{l}\text { Sex, age, weight, height, ethnicity, residency, } \\
\text { education, and occupation }\end{array}$ & $6-13$ \\
\hline \multicolumn{4}{|l|}{ SECTION B (case/control) } \\
\hline Phenotype & Self-administered & Skin type, eye/hair colour, freckles, nevi in childhood & $1-5$ \\
\hline History of sun and UVR exposure & Self-administered & Sun exposure and related habits, sunburns, lamps & $6-14$ \\
\hline Lifestyle habits & Self-administered & Vitamins, smoking & $15-18$ \\
\hline Section B Completion evaluation questions & Self-administered & Questionnaire evaluation & $19-20$ \\
\hline \multicolumn{4}{|l|}{ SECTION C (case/control) } \\
\hline Clinical examination & Physician/study nurse & SOLAR lentigines, nevi, actinic keratosis & $1-8$ \\
\hline Medical history/medications & Physician/study nurse & Non-cancer diagnoses, treatments, pregnancies, other cancers & $9-13$ \\
\hline Family history & Physician/study nurse & Melanoma and other cancers among relatives, genetic test & $14-16$ \\
\hline Section C Completion evaluation questions & Physician/study nurse & Questionnaire evaluation & $17-18$ \\
\hline \multicolumn{4}{|l|}{ SECTION D (case) } \\
\hline $\begin{array}{l}\text { Melanoma characteristics } \\
\text { (for each melanoma diagnosis) }\end{array}$ & Physician/study nurse & $\begin{array}{l}\text { Clinical presentation, number of melanomas, } \\
\text { date of diagnosis, site, histological type and } \\
\text { features, SLN* AJCC stage, mutation status }\end{array}$ & $1-13$ \\
\hline
\end{tabular}

*Sentinel Lymph Node Biopsy.

phenotyping is annotated and the date(s) of the updated phenotyping can also be recorded if additional patient interviewing or clinical evaluation is performed during follow-up. Subsection II includes questions about age, sex, weight and height, ethnicity, residency, education and occupation. The question about the place of residence is open-ended to capture all possible changes (and duration thereof). To facilitate categorization, geocoding is advisable. The question regarding history of occupation is also designed as open-ended and could also be supported by online tools or flash cards. The question about education has been harmonized to take into account country-specific differences. For this purpose, age categories defining educational levels have been set broadly to enable collection of the relevant data across different cohorts.

Section B is completed by the study subject. Its subsection I pertains to epidemiologic and clinical risk factors including skin type, eye colour, hair colour, freckling and nevi in childhood/ adolescence. To facilitate consistency in data reporting, the questions regarding the skin type are formulated to combine the subject's skin response to sun exposure with his/her ability to tan (Figure S1). The term 'skin colour' is not included in MelanoQ to avoid subjective assessment. Information on eye and hair colour has been merged into fewer categories (light: blue/green/ grey, medium: light brown/hazel and dark: dark brown/black, for eye colour; and red, blond, light brown, dark brown and black, for hair colour) to facilitate statistical analyses. Definition of freckles and solar lentigines is included to ensure proper identification of these lesions. Charts with images of eye and hair colour (Figures S2 and S3, respectively), as well as freckle and nevi density (Figures S4 and S5, respectively) have been included to standardize categorization and recording. Section $B$ also includes questions on occupational and recreational sun exposure, use of artificial tanning, sunburns and the use of sun protection methods (subsection II). Information on sun exposure and related behaviours also includes data on intermittent sun exposure (during childhood, adolescence and adulthood, as well as during a 10-year period prior to melanoma diagnosis). The number and age of occurrence $(<18$ years or $\geq 18$ years) of severe (grade 2 ) sunburns are also recorded, as is their site with respect to melanoma (where applicable). The use of sunscreen is categorized as never, $<50 \%$ or $>50 \%$ of the time during sun exposure, always, or not known, over four periods of life. Additional questions focus on lifestyle habits, specifically, vitamin intake and smoking history (subsection III).

Section C includes clinical examination, detailed medical history and family history of melanoma/other cancers for both cases and controls. This section is designed to be completed by a health care professional. The clinical examination part aims at recording the presence of multiple and/or clinically atypical melanocytic nevi, congenital melanocytic nevi, blue nevi, actinic keratoses and keratinocyte skin cancer KSC (basal cell carcinoma and squamous cell carcinoma) (subsection I). Nevi $>2 \mathrm{~mm}$ in size are recorded as a continuous variable by site and by laterality. Clinically, atypical nevi are defined as having a macular component in at least one part of the lesion (mandatory), and having at least 3 of the following criteria: $>5 \mathrm{~mm}$ diameter, colour variegation, irregular borders or erythema. ${ }^{22,23}$ Congenital nevi classified as medium-sized $(>1.5-19.9 \mathrm{~cm})$, large $(\geq 20 \mathrm{~cm})$, or giant $(\geq 40 \mathrm{~cm})$ are recorded as present or absent and their site is noted. The presence of actinic keratoses is also recorded 
specifying site and distribution and recommending the use of recently described scoring systems such as the AKASI or AK-FAS scores. $^{24,25}$ Information about the presence of KSC at the time of physical examination (subsection I) or of any history of previous KSC (subsection II) is also recorded, in the latter case with details on tumour type, number of tumours and time of appearance with respect to melanoma diagnosis. Additional questions in this section focus on medical history, reproductive history and medications (subsection II). Finally, subsection III collects information about family history of melanoma and other cancers. A positive family history of melanoma is considered relevant in the presence of $\geq 2$ first- to third-degree relatives affected by in situ or invasive melanoma. This definition takes into account the generally lower incidence of melanoma in the Mediterranean basin compared to other geographical regions, and the specific features of the Mediterranean populations. ${ }^{26}$ If possible, a family pedigree for each subject reporting a positive family history of melanoma should be added to the collected information.

Section $\mathrm{D}$ is the diagnostic section of the questionnaire designed for patients with a confirmed diagnosis of melanoma. It should be completed by a health care professional and aims to collect data on clinical presentation, including site of primary melanoma, Breslow thickness (and other major histopathologic features), American Joint Committee on Cancer (AJCC) stage, date of diagnosis and somatic mutational data (if available) (subsection I). All thirteen items should be recorded separately for each melanoma if the patient received multiple melanoma diagnoses. Notably, MelanoQ includes twelve items, which are marked as optional, that is to be collected only for the purpose of more detailed analyses.

We have tested MelanoQ in a total of 62 patients at five MelaNostrum centres in Italy, Spain, Greece. The time required for completion ranged from 22 to 60 minutes (average $40 \mathrm{~min}$ ). Although the completion time of the medical sections (C and D) was similar across physicians and centres (15-20 min), the selfadministered part (Section B) was more variable in duration (12-30 $\mathrm{min}$ ). In particular, questions $6-9$ and 11 in subsection II (Section B) required a longer time especially in more senior patients/controls or patients with lower educational background. When we added the assistance of an interviewer for these people, the completion time decreased and the quality of the information increased substantially. We thus have added a separate evaluation section (questions \#19, 20 in Section B and questions $\# 17,18$ in Section C) that enables to score the degree of difficulty in responding to the self-administered questions in section $\mathrm{B}$ as well as to the phenotypical/clinical information in Section $\mathrm{C}$ by the physician/nurse.

\section{Discussion}

Upon establishment of the MelaNostrum Consortium, one of the major issues that members had to deal with (and solve) was the incomplete or heterogeneous recording of the information on melanoma patients' specific risk factors, especially skin phototype, sun exposure patterns and nevus assessment, collected at each participating centre. We report herein on the development of a standardized tool that expedites collection of epidemiologic and clinical data on CM from diverse case-control datasets to allow association analyses across different centres. MelanoQ also provides a template for data compilation for prospective studies, clinical research projects or trials. Even though it has been designed more as a research tool for epidemiologic studies and may not be suitable for completion in the context of the rapid pace of daily clinical practice, it may also serve as database for collection of pertinent clinico-pathological information on newly diagnosed patients in melanoma centres.

Previous questionnaires for melanoma studies include the Q-skin, ${ }^{27}$ the Sun Exposure Behaviour Inventory (SEBI), ${ }^{17}$ and the Sun Exposure and Protection Index (SEPI), ${ }^{18}$ among others. ${ }^{19-21}$ The Q-skin questionnaire is considered one of the most complete tools. However, its test-retest reliability for the measurement of phenotypic characteristics, sun exposure and nevus-related variables was not high. ${ }^{27}$ Similarly, the repeatability of the SEBI instrument, which was limited in scope, since it was designed to assess current and prior sun exposure, was found to be only satisfactory. ${ }^{17}$ MelanoQ has the advantage of allowing collection of data on individual risk assessment for epidemiologic purposes, but it can also be used as an entry tool by clinical centres managing melanoma patients. At the same time, although certain items in MelanoQ should be collected by clinicians or specialized personnel, several parts can be self-administered.

In designing this data collection tool, we first identified the most relevant questions to be used for investigating disease aetiology and progression. We also strove to clearly formulate simple questions for the lay public and precoded the responses for statistical analyses. In order to facilitate data collection, we added charts for eye colour, hair colour, freckling and nevus density, lists of ethnicities, examples of outdoor occupations and recreational activities and online tools for residency and occupation recording. Finally, we added an evaluation section to identify the most challenging self-administered questions that may need follow-up by the investigators.

An important part of MelanoQ relates to the collection of information on pigmentation characteristics. Fair skin type (Fitzpatrick I or II), ${ }^{28}$ red or blond hair and the presence of freckles have all been associated with a higher risk of melanoma. ${ }^{2915,16,30-34}$ Eye colour has also shown an association with melanoma risk in numerous studies. ${ }^{15,16,35-38}$ In MelanoQ, we have reproduced the categorization of pigmentary features that has been used for melanoma studies, with a larger spectrum of phototypes for subjects with relatively dark complexion, like those in the Mediterranean population. 
The importance of recreational/intermittent sun exposure for melanoma risk has been well documented, while the role of occupational/continuous sun exposure remains unclear. ${ }^{13,39} \mathrm{~A}$ standardized way of recording these two types of exposure by self-administered questionnaires has been proposed, which aims at capturing the amount of exposure time during daylight hours for both recreational and occupational activities. ${ }^{40}$ Previous studies with existing standardized tools have shown reasonable reproducibility although the validity of the responses is unclear. $^{41,42}$ The items specifically related to sun exposure in MelanoQ have been designed as simple, closed-ended questions. For instance, for intermittent sun exposure, a short description is included (i.e. in spring and summer time) and the case/control subject is requested to provide an answer for four predesignated time periods (section B-II, question 8). We also included questions to record sunscreen use and sun protection methods over time. $^{43,44}$

The identification and recording of common melanocytic and atypical nevi-two well-established risk factors associated with a high risk of melanoma-proved to be one of the most difficult tasks in the epidemiologic investigation of melanoma. ${ }^{12,45}$ Although the issues associated with the identification of melanocytic lesions were partially addressed by the implementation of dermoscopy in the daily clinical practice, the need for a harmonized way to record nevus counts still exists. Before deciding how to collect nevi data, the MelaNostrum clinicians discussed the diagnosis and measurement criteria, reviewed the abundant literature on the topic ${ }^{12,22,23,45-47}$ and addressed the inter-observer differences in nevus count. ${ }^{48,49}$ The group also performed joint patient examinations to reach a consensus on how to report, easily and precisely, nevus counts and relevant risk factors. The questions in MelanoQ are in agreement with the International Agency for Research on Cancer (IARC) protocol for the identification and recording of nevi, ${ }^{47,50}$ which has been implemented in previous studies. ${ }^{51-54}$ Items that can help one to collect information on the presence of congenital nevi sized $>1.5 \mathrm{~cm}$, and particularly those with diameter $\geq 20 \mathrm{~cm}$ or giant nevi, which are considered at risk of malignant transformation, have also been included in our tool. ${ }^{55-59}$

Section D of MelanoQ contains highly structured questions regarding patient melanoma diagnosis, including anatomical site of the lesion, tumour histopathologic features and tumour mutational status information (if applicable). The completeness of the clinical and histopathological data collected by the SEER registries and their impact on the transition from the AJCC 6th to the 7 th edition with regards to stage distribution has been previously assessed. ${ }^{60}$ It was reported that about $10 \%$ of cases were coded as unknown for measured thickness, ulceration and lymph node metastasis, suggesting the need for enhanced data capture for staging purposes. ${ }^{60}$ We created a specific checklist for melanoma diagnosis data collection to reduce the possibility of incomplete data recording as a result of human error.
MelanoQ can be enriched with additional items to generate hypothesis-driven questions and to include new information that may prove to be associated with melanoma risk in the future. Our tool may expedite ongoing efforts to compile highquality data for pooled analyses or meta-analyses and offer a solid base for the design of clinical, epidemiologic and translational studies on melanoma. We have already tested MelanoQ across different clinical centres, languages and cultures in Greece, Italy and Spain and plan, as a future step, to verify its validity and repeatability by applying it to other populations.

\section{Acknowledgements}

Dr. Ghiorzo wishes to thank Dr. Giulia Ciccarese for contributing to the development of the questionnaire. Dr. De Nicolo wishes to thank Prof. Mauro S. A. Alaibac (Department of Medicine, University of Padua) and his team for testing the questionnaire. Dr. Puig would like to acknowledge all members from the Melanoma Unit of Hospital Clínic of Barcelona: Paula Aguilera, Beatriz Alejo, Llucia Alos, Celia Badenas, Alicia Barreiro, Abel Caño, Cristina Carrera, Mireia Domínguez, Natalia Espinosa, Daniel Gabriel, Pol Gimenez-Xavier, Pablo Iglesias, Josep Malvehy, Judit Mateu, Maria E Moliner, Miriam Potrony, Joan Anton Puig-Butille, Carolina Sanchez, Gemma Tell and Oriol Yelamos.

\section{References}

1 Holman DM, Freeman MB, Shoemaker ML. Trends in melanoma incidence among non-Hispanic whites in the United States, 2005 to 2014. JAMA Dermatol 2018; 154: 361-362.

2 Coviello V, Buzzoni C, Fusco M et al. Survival of cancer patients in Italy. Epidemiol Prev 2017; 41(2 Suppl 1): 1-244.

3 Busco S, Buzzoni C, Mallone S et al. Italian cancer figures-Report 2015: The burden of rare cancers in Italy. Epidemiol Prev 2016; 40(1 Suppl 2): $1-120$.

4 Puig S, Marcoval J, Paradelo C et al. Melanoma incidence increases in the elderly of Catalonia but not in the younger population: effect of prevention or consequence of immigration? Acta Dermato-venereologica 2015; 95: 422-426.

5 Leiter U, Eigentler T, Garbe C. Epidemiology of skin cancer. Adv Exp Med Biol 2014; 810: 120-140.

6 Ferlay J, Soerjomataram I, Dikshit R et al. Cancer incidence and mortality worldwide: sources, methods and major patterns in GLOBOCAN 2012. Int J Cancer 2015; 136: E359-E386.

7 Sacchetto L, Zanetti R, Comber H et al. Trends in incidence of thick, thin and in situ melanoma in Europe. Eur J Cancer 2018; 92: 108-118.

8 Wu YP, Kohlmann W, Curtin K et al. Melanoma risk assessment based on relatives' age at diagnosis. Cancer Causes Control 2017; 29: 193-199.

9 Conic RZ, Cabrera CI, Khorana AA, Gastman BR. Determination of the impact of melanoma surgical timing on survival using the National Cancer Database. J Am Acad Dermatol 2018; 78: 40-46.e47.

10 Pampena R, Kyrgidis A, Lallas A, Moscarella E, Argenziano G, Longo C. A meta-analysis of nevus-associated melanoma: Prevalence and practical implications. J Am Acad Dermatol 2017; 77: 938-945.e934.

11 Shin TM, Shaikh WR, Etzkorn JR, et al. Clinical and pathologic factors associated with subclinical spread of invasive melanoma. J Am Acad Dermatol 2017; 76: 714-721.

12 Gandini S, Sera F, Cattaruzza MS et al. Meta-analysis of risk factors for cutaneous melanoma: I. Common and atypical naevi. Eur J Cancer 2005; 41: $28-44$. 
13 Gandini S, Sera F, Cattaruzza MS et al. Meta-analysis of risk factors for cutaneous melanoma: II. Sun exposure. Eur J Cancer 2005; 41: 45-60.

14 Gandini S, Sera F, Cattaruzza MS et al. Meta-analysis of risk factors for cutaneous melanoma: III. Family history, actinic damage and phenotypic factors. Eur J Cancer 2005; 41: 2040-2059.

15 Nijsten T, Leys C, Verbruggen K et al. Case-control study to identify melanoma risk factors in the Belgian population: the significance of clinical examination. J Eur Acad Dermatol Venereol 2005; 19: 332-339.

16 Nikolaou VA, Sypsa V, Stefanaki I et al. Risk associations of melanoma in a Southern European population: results of a case/control study. Cancer Causes Control 2008; 19: 671-679.

17 Jennings L, Karia PS, Jambusaria-Pahlajani A, Whalen FM, Schmults CD. The Sun Exposure and Behaviour Inventory (SEBI): validation of an instrument to assess sun exposure and sun protective practices. J Eur Acad Dermatol Venereol 2013; 27: 706-715.

18 Detert H, Hedlund S, Anderson CD et al. Validation of sun exposure and protection index (SEPI) for estimation of sun habits. Cancer Epidemiol 2015; 39: 986-993.

19 Reeder AI, Hammond VA, Gray AR. Questionnaire items to assess skin color and erythemal sensitivity: reliability, validity, and "the dark shift". Cancer Epidemiol, Biomarkers Prev 2010; 19: 1167-1173.

20 Tacke J, Dietrich J, Steinebrunner B, Reifferscheid A. Assessment of a new questionnaire for self-reported sun sensitivity in an occupational skin cancer screening program. BMC Dermatol 2008; 8: 4

21 Falk M, Anderson CD. Measuring sun exposure habits and sun protection behaviour using a comprehensive scoring instrument-an illustration of a possible model based on Likert scale scorings and on estimation of readiness to increase sun protection. Cancer Epidemiol 2012; 36: e265-e269.

22 Tucker MA, Halpern A, Holly EA et al. Clinically recognized dysplastic nevi. A central risk factor for cutaneous melanoma. JAMA 1997; 277: 1439-1444.

23 Duffy K, Grossman D. The dysplastic nevus: from historical perspective to management in the modern era: part I. Historical, histologic, and clinical aspects. J Am Acad Dermatol 2012; 67: 1.e1-16; quiz 17-18.

24 Dirschka T, Pellacani G, Micali G et al. A proposed scoring system for assessing the severity of actinic keratosis on the head: actinic keratosis area and severity index. J Eur Acad Dermatol Venereol 2017; 31: 1295-1302.

25 Dreno B, Cerio R, Dirschka T et al. A novel actinic keratosis field assessment scale for grading actinic keratosis disease severity. Acta DermatoVenereologica 2017; 97: 1108-1113.

26 Soura E, Eliades PJ, Shannon K, Stratigos AJ, Tsao H. Hereditary melanoma: Update on syndromes and management: Genetics of familial atypical multiple mole melanoma syndrome. J Am Acad Dermatol 2016; 74: 395-407.

27 Morze CJ, Olsen CM, Perry SL et al. Good test-retest reproducibility for an instrument to capture self-reported melanoma risk factors. J Clin Epidemiol 2012; 65: 1329-1336.

28 Fitzpatrick TB. The validity and practicality of sun-reactive skin types I through VI. Arch Dermatol 1988; 124: 869-871.

29 Olsen CM, Carroll HJ, Whiteman DC. Estimating the attributable fraction for melanoma: a meta-analysis of pigmentary characteristics and freckling. Int J Cancer 2010; 127: 2430-2445.

30 Lasithiotakis K, Kruger-Krasagakis S, Ioannidou D, Pediaditis I, Tosca A. Epidemiological differences for cutaneous melanoma in a relatively darkskinned Caucasian population with chronic sun exposure. Eur J Cancer 2004; 40: 2502-2507.

31 Fargnoli MC, Piccolo D, Altobelli E, Formicone F, Chimenti S, Peris K. Constitutional and environmental risk factors for cutaneous melanoma in an Italian population. A case-control study. Melanoma Res 2004; 14: 151-157.

32 Naldi L, Lorenzo Imberti G, Parazzini F, Gallus S, La Vecchia C. Pigmentary traits, modalities of sun reaction, history of sunburns, and melanocytic nevi as risk factors for cutaneous malignant melanoma in the Italian population: results of a collaborative case-control study. Cancer 2000; 88: 2703-2710.
33 Rodenas JM, Delgado-Rodriguez M, Herranz MT, Tercedor J, Serrano S. Sun exposure, pigmentary traits, and risk of cutaneous malignant melanoma: a case-control study in a Mediterranean population. Cancer Causes Control 1996; 7: 275-283.

34 Ghiorzo P, Bonelli L, Pastorino L et al. MC1R variation and melanoma risk in relation to host/clinical and environmental factors in CDKN2A positive and negative melanoma patients. Exp Dermatol 2012; 21 : 718-720.

35 Veierod MB, Weiderpass E, Thorn $\mathrm{M}$ et al. A prospective study of pigmentation, sun exposure, and risk of cutaneous malignant melanoma in women. J Natl Cancer Inst 2003; 95: 1530-1538.

36 Titus-Ernstoff L, Perry AE, Spencer SK, Gibson JJ, Cole BF, Ernstoff MS Pigmentary characteristics and moles in relation to melanoma risk. Int $J$ Cancer 2005; 116: 144-149.

37 Lock-Andersen J, Drzewiecki KT, Wulf HC. Eye and hair colour, skin type and constitutive skin pigmentation as risk factors for basal cell carcinoma and cutaneous malignant melanoma. A Danish case-control study. Acta Dermato-venereologica 1999; 79: 74-80.

38 Zanetti R, Rosso S, Martinez C et al. Comparison of risk patterns in carcinoma and melanoma of the skin in men: a multi-centre case-case-control study. Br J Cancer 2006; 94: 743-751.

39 Chang YM, Barrett JH, Bishop DT et al. Sun exposure and melanoma risk at different latitudes: a pooled analysis of 5700 cases and 7216 controls. Int J Epidemiol 2009; 38: 814-830.

40 Karagas MR, Zens MS, Nelson HH et al. Measures of cumulative exposure from a standardized sun exposure history questionnaire: a comparison with histologic assessment of solar skin damage. Am J Epidemiol 2007; 165: 719-726.

41 Kricker A, Armstrong BK, English DR, Heenan PJ. Does intermittent sun exposure cause basal cell carcinoma? a case-control study in Western Australia. Int J Cancer 1995; 60: 489-494.

42 Gallagher RP, Hill GB, Bajdik CD et al. Sunlight exposure, pigmentation factors, and risk of nonmelanocytic skin cancer. II. Squamous cell carcinoma. Arch Dermatol 1995; 131: 164-169.

43 Ghiasvand R, Weiderpass E, Green AC, Lund E, Veierod MB. Sunscreen use and subsequent melanoma risk: a population-based cohort study. J Clin Oncol 2016; 34: 3976-3983.

44 Autier P, Boniol M, Dore JF. Sunscreen use and increased duration of intentional sun exposure: still a burning issue. Int J Cancer 2007; 121: $1-5$.

45 Chang YM, Newton-Bishop JA, Bishop DT et al. A pooled analysis of melanocytic nevus phenotype and the risk of cutaneous melanoma at different latitudes. Int J Cancer 2009; 124: 420-428.

46 Tsao H, Sober A. Atypical melanocytic nevi. In: Orioti-Parreiras IM, ed. Fitzpatrick's Dermatology in General Medicine. Vol 1. New York, NY: McGraw-Hill; 2003:906-916.

47 Gallagher RP, McLean DI. The epidemiology of acquired melanocytic nevi. A brief review. Dermatol Clin 1995; 13: 595-603.

48 English JS, Swerdlow AJ, MacKie RM et al. Relation between phenotype and banal melanocytic naevi. BMJ (Clin Res Ed) 1987; 294: 152-154.

49 MacKie RM, English J, Aitchison TC, Fitzsimons CP, Wilson P. The number and distribution of benign pigmented moles (melanocytic naevi) in a healthy British population. Br J Dermatol 1985; 113: 167-174.

50 English D, MacLennan R, Rivers JK, Kelly J, Amstrong BK. Epidemiological Studies of Melanocytic Naevi: Protocol for Identifying and Recording Naevi. Lyon: International Agency for Research on Cancer 1990.

51 Bataille V, Bishop JA, Sasieni P et al. Risk of cutaneous melanoma in relation to the numbers, types and sites of naevi: a case-control study. $\mathrm{Br} \mathrm{J}$ Cancer 1996; 73: 1605-1611.

52 Mossner R, Anders N, Konig IR et al. Variations of the melanocortin-1 receptor and the glutathione-S transferase $\mathrm{T} 1$ and $\mathrm{M} 1$ genes in cutaneous malignant melanoma. Arch Dermatol Res 2007; 298: 371-379.

53 Berwick M, Begg CB, Fine JA, Roush GC, Barnhill RL. Screening for cutaneous melanoma by skin self-examination. J Natl Cancer Inst 1996; 88: $17-23$. 
54 Kanetsky PA, Holmes R, Walker A et al. Interaction of glutathione S-transferase M1 and T1 genotypes and malignant melanoma. Cancer Epidemiol Biomark Prev 2001; 10: 509-513.

55 Vourc'h-Jourdain M, Martin L, Barbarot S. Large congenital melanocytic nevi: therapeutic management and melanoma risk: a systematic review. J Am Acad Dermatol 2013; 68: 493-498.e491-414.

56 Alikhan A, Ibrahimi OA, Eisen DB. Congenital melanocytic nevi: where are we now? Part I. Clinical presentation, epidemiology, pathogenesis, histology, malignant transformation, and neurocutaneous melanosis. J Am Acad Dermatol 2012; 67: 495.e491-417; quiz 512-494.

57 Price HN, O’Haver J, Marghoob A, Badger K, Etchevers H, Krengel S. Practical application of the new classification scheme for congenital melanocytic nevi. Pediatr Dermatol 2015; 32: 23-27.

58 Tannous ZS, Mihm MC Jr, Sober AJ, Duncan LM. Congenital melanocytic nevi: clinical and histopathologic features, risk of melanoma, and clinical management. J Am Acad Dermatol 2005; 52: 197-203.

59 Kinsler VA, O'Hare P, Bulstrode $\mathrm{N}$ et al. Melanoma in congenital melanocytic naevi. Br J Dermatol 2017; 176: 1131-1143.

60 Kosary CL, Altekruse SF, Ruhl J, Lee R, Dickie L. Clinical and prognostic factors for melanoma of the skin using SEER registries: collaborative stage data collection system, version 1 and version 2. Cancer 2014; 120(Suppl 23): 3807-3814.

\section{Supporting information}

Additional supporting information may be found online in the Supporting Information section at the end of the article.

Figure S1. Tanning ability chart.

Figure S2. Eye colour chart.

Figure S3. Hair colour chart.

Figure S4. Freckle density chart (Chart adapted by the Q-questionnaire tool - by permission).

Figure S5. Nevus density chart.

Table S1. Centres and members of the MelaNostrum Consortium.

Table S2. The complete MelaNostrum questionnaire.

Appendix S1. List of ethnicities. 